\title{
Physics with Open Charm Mesons at $\overline{\mathbf{P}}$ ANDA
}

\author{
A. Sokolov*, A. Gillitzer* and J. Ritman* \\ *IKP I, FZ Jülich, Germany
}

\begin{abstract}
We present a study of the physics performance of the $\overline{\mathbf{P} A N D A}$ detector based on MonteCarlo simulations. The possibility to measure $D, D^{*}$ and $D_{s J}^{*}(2317)$ mesons has been investigated. A reliable detection of open charm decay products of charmonium decays and sufficient level of background suppression have been demonstrated.
\end{abstract}

Keywords: PANDA; HESR; charmed mesons; threshold production.

PACS: 13.25 .Es

\section{INTRODUCTION}

The study of the charmonium spectrum above the $D \bar{D}$ breakup threshold at $3.73 \mathrm{GeV} / \mathrm{c}^{2}$ is an important part of the physics program of the forthcoming $\overline{\mathbf{P}}$ ANDA experiment at FAIR/Darmstadt [1]. The scope of experiments include a confirmation of the higher vector states at 4040,4160 and $4415 \mathrm{MeV} / \mathrm{c}$ together with systematic investigation of the $D_{s J}$ and $D_{s}^{*}$ mesons. In addition, there are also several hypotheses which can be tested using the unprecedented beam quality delivered by the HESR facility. For example the confirmation of an interpretation of the X(3872) state, recently discovered by the BELLE collaboration, as a $D^{0} \overline{D^{* 0}}$ molecule requires a precision measurement of all ${ }^{1} D$ and ${ }^{3} D$ states. Another kind of experiment is threshold production of the recently discovered $D_{s J}^{*}$ mesons, which will precisely measure their masses and decay widths, with the goal to strongly constrain models of their structure.

\section{HIGHER CHARMONIA}

\section{Physics background}

Above the $D \bar{D}$ breakup threshold at $3.73 \mathrm{GeV} / \mathrm{c}^{2}$ the charmonium spectrum is poorly known (see Fig. 1, left) since $e^{+} e^{-}$experiments have only measured $R=\sigma\left(e^{+} e^{-} \rightarrow\right.$ hadrons $) / \sigma\left(e^{+} e^{-} \rightarrow \mu^{+} \mu^{-}\right)$in large energy steps. Here, rather washed-out structures were observed. The latest, much more accurate measurements by BES [2] do not confirm the sharp states reported by [3]. It is an open question whether the higher vector states at 4040,4160 , and $4415 \mathrm{MeV} / \mathrm{c}^{2}$ are realistic.

Several theories exist explaining the relative narrowness of the $\psi(4040)$ state. It can be a $\mathrm{D}^{*} \overline{\mathrm{D}^{*}}$ molecule [4]. According to an other idea [5] the $\psi(4040)$ and $\psi(4160)$ are strong mixtures of ground state hybrid charmonium at $\sim 4.1 \mathrm{GeV} / \mathrm{c}^{2}$ and $\psi(3 \mathrm{~S})$ of conventional charmonium. Both theories predict different decay patterns for both states.

It is of critical importance to investigate this mass region in small energy steps. 


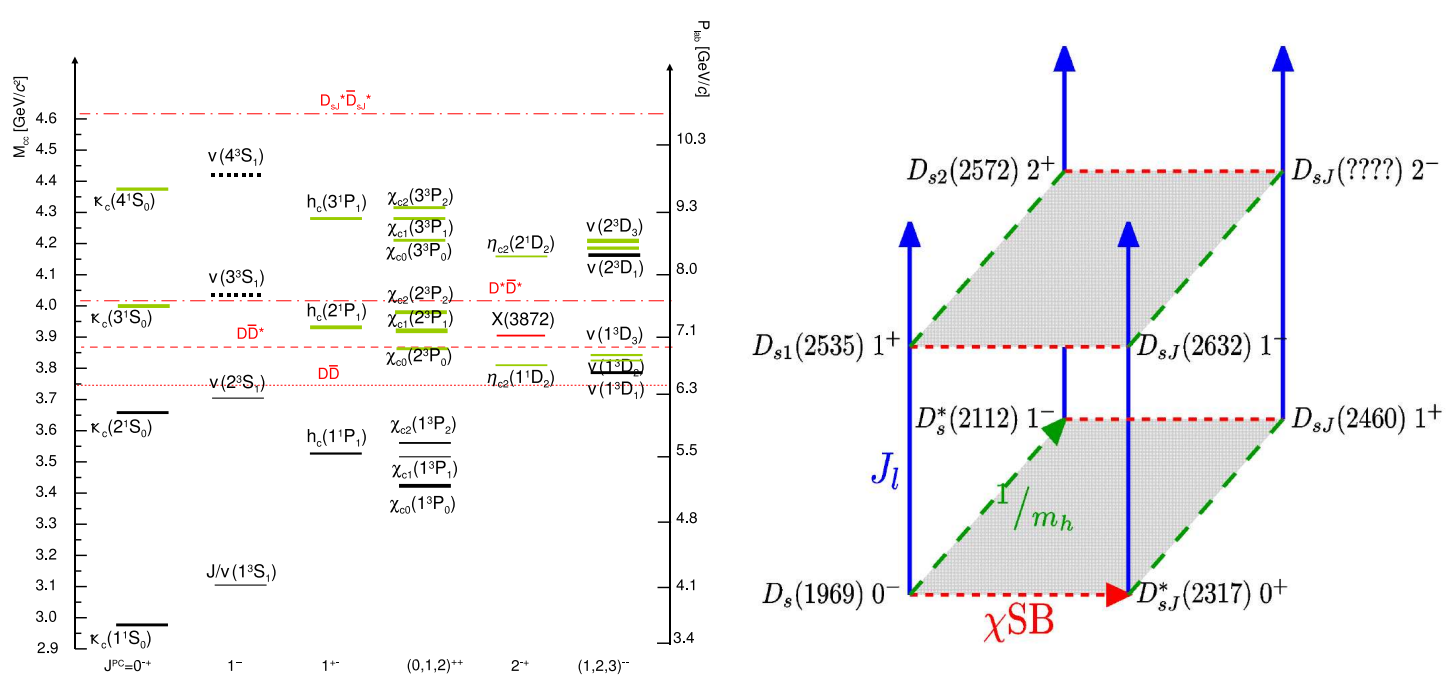

FIGURE 1. The charmonium spectrum and schematic classification of chiral doublers are shown on the left and right figures, respectively.

\section{$D^{+} D^{-}$and $D^{*+} D^{*-}$ pair selection}

Monte Carlo simulations have been done to demonstrate that the proposed $\overline{\mathbf{P}}$ ANDA detector can fulfill the physics requirements.

The performance of the $D^{ \pm} \rightarrow K^{\mp} \pi^{ \pm} \pi^{ \pm}$selection has been studied by simulating 1000 events with exactly one $D$ meson each. These mesons were generated with $3.3 \mathrm{GeV} / \mathrm{c}^{2}$ momentum, which corresponds to half the beam momentum for a formation experiment of the $\psi(3770)$. The event generator was modified to force the decay in every event to occur at the same location $(0.1,0.1,0.4) \mathrm{mm}$ with respect to the nominal target position.

The resolution for the three particle tracks vertex is presented in Fig. 2 (left) and Fig. 2 (right). In Fig. 2 (left) the transverse distance of the vertex to the beam axis is given. The mean location of $0.152 \mathrm{~mm}$ is in a good agreement with the expected value of $\sqrt{2} \times 0.1 \mathrm{~mm}=0.141 \mathrm{~mm}$. The resolution of the vertex is about $50 \mu \mathrm{m}$ in the transverse and $50 \mu \mathrm{m}$ in the longitudinal directions.

The overall $\overline{\mathbf{P}}$ ANDA detector performance have been studied for the reactions $\bar{p} p \rightarrow \psi(3770) \rightarrow D^{+} D^{-} \rightarrow K^{-} \pi^{+} \pi^{+}+c . c$. and $\bar{p} p \rightarrow \psi(4040) \rightarrow D^{*+} D^{*-} \rightarrow$ $D^{0} \pi^{+} D^{-} \pi^{-} \rightarrow K^{-} \pi^{+} \pi^{+}+c . c$. . In Fig. 3 the invariant mass distributions for the $\psi(3770)$ and $\psi(4040)$ are shown reconstructed from 50000 signal events without additional background events. The invariant mass resolutions $\sigma$ and the reconstruction efficiency $\varepsilon$ are found to be:

$$
\begin{gathered}
\sigma_{\psi(3770)}=10 \mathrm{MeV} / \mathrm{c}^{2} \text { and } \varepsilon_{\psi(3770)} \simeq 4.2 \% \\
\sigma_{\psi(4040)}=19 \mathrm{MeV} / \mathrm{c}^{2} \text { and } \varepsilon_{\psi(4040)} \simeq 10.4 \%
\end{gathered}
$$



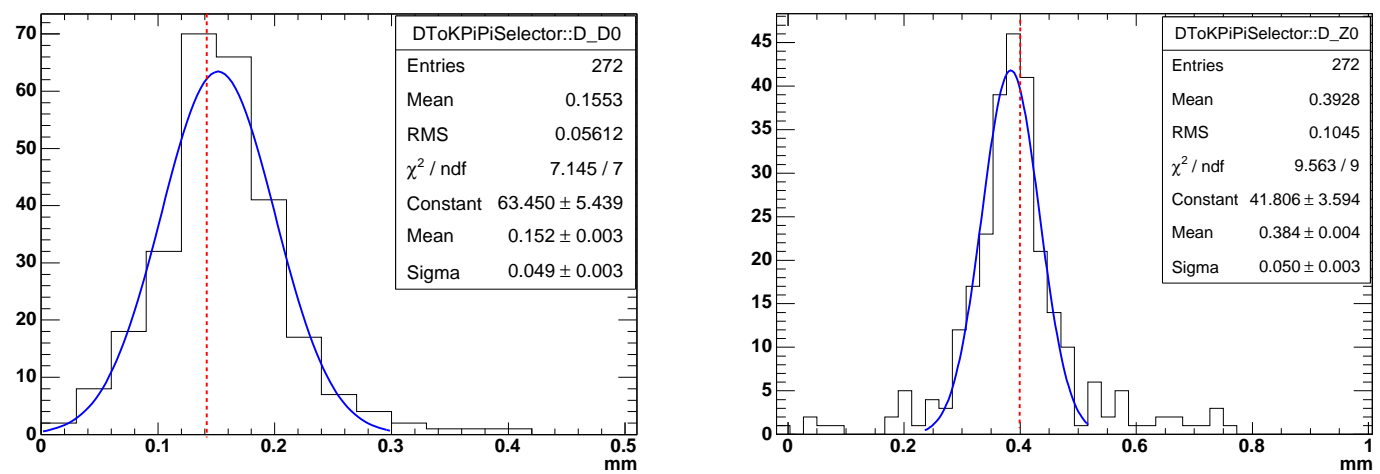

FIGURE 2. Distribution of the transverse and longitudinal distances of the vertex location to the primary vertex position of $D$ mesons are shown on the left and right figures, respectively. The expected locations are shown by the dashed lines. See text for more explanations.
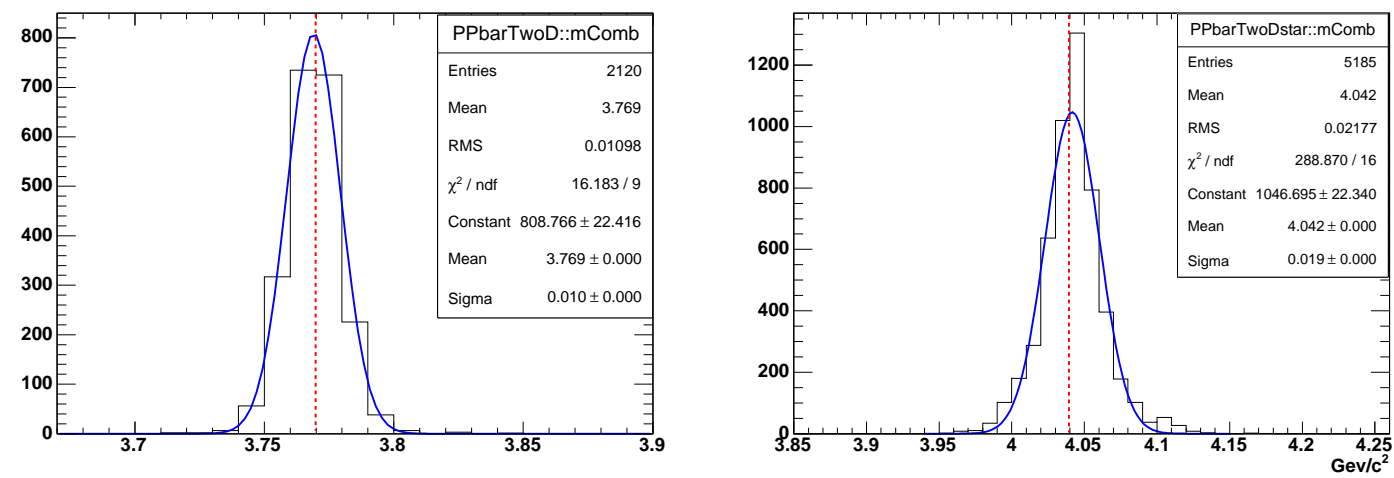

FIGURE 3. The reconstructed $\psi(3770)$ and $\psi(4040)$ invariant masses are shown on the left and right figures, respectively. The expected peaks positions are marked by the dashed lines.

From the analysis of a large sample of background events a signal to backgroud ratio of $\sim 3$ for the $\psi(3770)$ state have been determined. For the $\psi(4040)$ only lower limit of $1.27 \times 10^{-2}$ has been determined.

\section{THRESHOLD PRODUCTION OF $D_{S J}^{*}(2317)$}

The recent observation by the BABAR collaboration of a narrow $D_{S J}^{*}(2317)^{+}$mesonic state [6] greatly stimulates the interest to the open charm spectroscopy. This observation was confirmed by CLEO [7], which also noticed a second $D_{S J}(2463)^{+}$resonance. Finally, both states were confirmed by Belle [8].

The $D_{S J}^{*}(2317)^{+}$state was identified as a scalar $c \bar{s}$ system, but it has a significantly smaller mass and width than expected (e.g. [9]). Many theories have appeared after that discovery explaining the unexpected properties of $D_{s J}^{*}(2317)^{+}$(see [10] or [11]). For example, the chiral doubling model introduces the chiral partners to four already known charm-strange mesonic states, i.e. $D_{s}(1969), D_{s}^{*}(2112), D_{s 1}(2536)$ and $D_{s 2}(2573)$. The 
schematic of the chiral doubling theory is shown on Fig. 1, right. The "left plaquette" of the cube represents standard charm-strange mesons, whereas the "right plaquette" stands for chiral doublers. Similar patterns can be applied also for the non-strange charmed mesons.

Predictions for the $D_{S J}^{*}(2317)^{+}$width range from 10 to $\sim 200 \mathrm{keV}$ and can give a good hint on its internal structure. The existing $e^{+} e^{-}$-colliders can't provide the width measurement with sufficient precision. The upper limit on the visible width, settled by the BABAR, is $\Gamma_{\text {exp }}<4.6 \mathrm{MeV}$. That problem can be solved by the $\overline{\mathbf{P}}$ ANDA experiment by measuring the excitation function of $\bar{p} p \rightarrow D_{S J}^{*}(2317)^{+} D_{s J}^{*}(2317)^{-}$close to the threshold.

The cross section behavior in the vicinity of the threshold depends on the beam momentum spread as it shown on Fig. 4. The invariant mass distribution for the
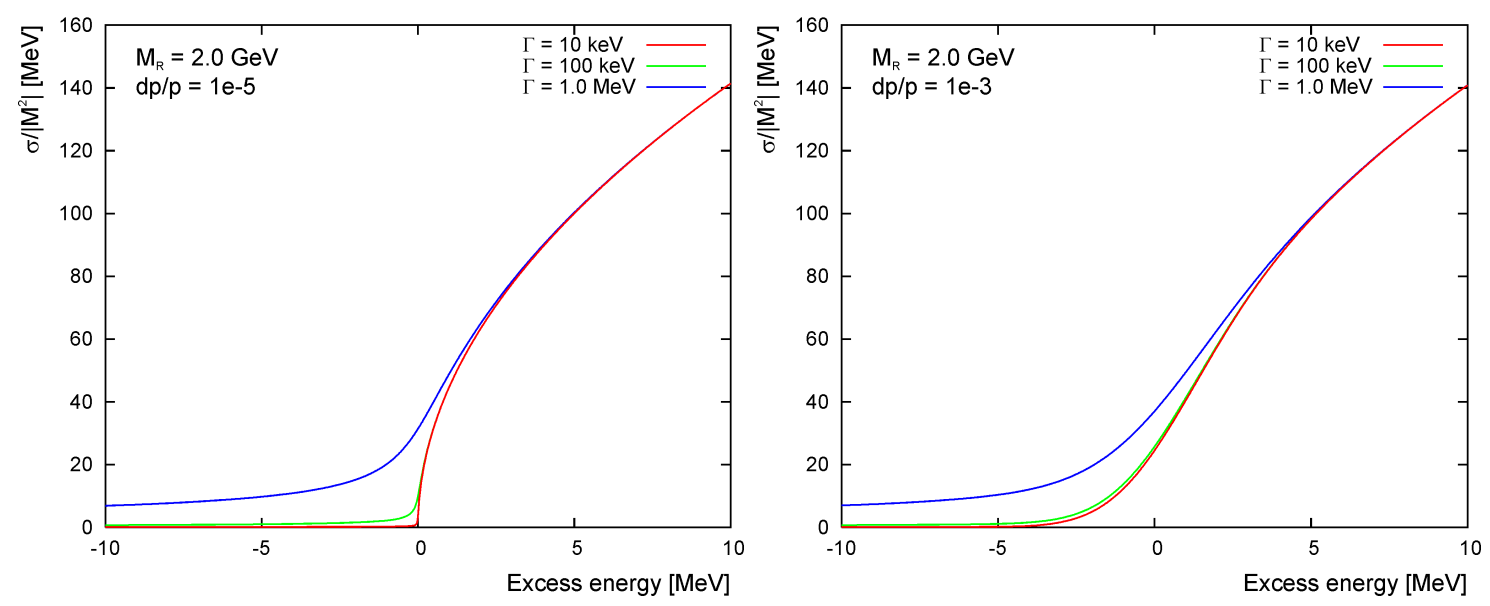

FIGURE 4. Energy dependence of $\bar{p} p \rightarrow D_{s J}^{*}(2317)^{+} D_{s J}^{*}(2317)^{-}$cross section for $10^{-5}$ (left) and $10^{-3}$ (right) relative momentum spread of the antiproton beam. The figures are taken from [12].

$D_{s J}^{*}(2317)^{+} D_{s J}^{*}(2317)^{-}$pairs is shown on Fig. 5 for 50000 signal events without background. The invariant mass resolution $\sigma$ and the reconstruction efficiency $\varepsilon$ are found to be:

$$
\sigma_{D_{s J}^{*+} D_{s J}^{*-}}=24 \mathrm{MeV} / \mathrm{c}^{2} \text { and } \varepsilon_{D_{s J}^{*+} D_{s J}^{*-}} \simeq 4.0 \%
$$

\section{CONCLUSIONS}

The simulations of the three benchmark reactions $\bar{p} p \rightarrow \psi(3770), \bar{p} p \rightarrow \psi(4040)$ and $\bar{p} p \rightarrow D_{s J}^{*}(2317)^{+} D_{s J}^{*}(2317)^{-}$show the capability of the $\overline{\mathbf{P}}$ ANDA detector to select the signals of these reactions from background. The reconstruction efficiencies for the different types of $D$ and $D_{s}$ mesons are shown in Table 1. A reasonable good signal to background ratio has been obtained despite the very small initial ratio of the signal/background of $\sim 10^{-8}$.

The detector structure is not yet fixed, so one can expect the further improvements in the invariant mass resolution and background rejection power. Nevertheless the results 


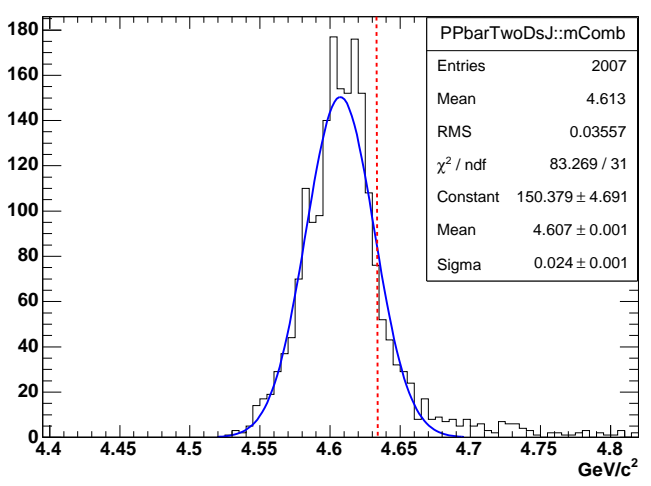

FIGURE 5. Reconstructed $D_{S J}^{*}(2317)^{+} D_{S J}^{*}(2317)^{-}$invariant mass. The expected peak position is shown by the dashed line.

TABLE 1. Reconstruction efficiency of $D$ and $D_{s}$ mesons.

\begin{tabular}{lcc}
\hline \multicolumn{1}{c}{ Decay } & Branching, \% & Efficiency, \% \\
\hline$D^{ \pm} \rightarrow K^{\mp} \pi^{ \pm} \pi^{ \pm}$ & 9.2 & 27 \\
$D^{0} \rightarrow K^{\mp} \pi^{ \pm}$ & 3.8 & 31 \\
$D^{0} \rightarrow K^{\mp} \pi^{ \pm} \pi^{\mp} \pi^{ \pm}$ & 7.5 & 20 \\
$D_{s}^{ \pm} \rightarrow K^{\mp} K^{ \pm} \pi^{ \pm}$ & 4.4 & $30^{*}$ \\
\hline
\end{tabular}

* Identification of only one kaon was required.

already look promising now and show that the $\mathbf{P}$ ANDA detector meets the physics requirement.

\section{ACKNOWLEDGMENTS}

This talk is based on the work done in preparation to the publication of PANDA Technical Progress Report [1]. We wish to express here our gratitude to Bertram Kopf, Mattias Steinke, Alexander Golischewski, Jan Zhong and many others members of $\overline{\mathbf{P}}$ ANDA Simulation group which help to organize the large scale production of MonteCarlo simulations.

\section{REFERENCES}

1. M. Kotulla and others, "Technical Progress Report," Panda Collaboration, Facility for Antiproton and Ion Research, Darmstadt, 2005.

2. J.Z. Bai and others, Phys. Rev. D, 57, 3854 (1998).

3. L. Antoniazzi and others, Phys. Rev. D, 50, 4258 (1994).

4. L. Okun, $M V^{\prime} 77,1977$.

5. F.E. Close and P.R. Page, Phys. Lett. B, 366, 323-328 (1996).

6. BABAR Coll., B. Aubert et al., hep-ex/0304021.

7. CLEO Coll., D. Besson et al., hep-ex/0305017, hep-ex/0305100. 
8. BELLE Coll., P. Krokovny et al., hep-ex/0308019.

9. M.A. Nowak, M. Rho and I. Zaled, Phys. Rev. D, 48, 4370 (1993).

10. M.A. Nowak, hep-ph/0311154, 2003.

11. Eef van Beveren and G. Rupp, hep-ph/0306155, 2004

12. A. Gillitzer, "How to measure sub-MeV width", XII. PANDA Collaboration Meeting, 2005. 